\title{
HAVING A SMALL WEIGHT IS DETERMINED BY THE SMALL SUBSPACES
}

\section{A. HANAL AND I. JUHÁSZ}

\begin{abstract}
We show that for every cardinal $\kappa>\omega$ and an arbitrary topological space $X$ if we have $w(Y)<\kappa$ whenever $Y \subset X$ and $|Y|<\kappa$ then $w(X)<\kappa$ as well. M. G. Tkacenko proved this for $T_{3}$ spaces in [2]. We also prove an analogous statement for the $\pi$-weight if $\alpha$ is regular.
\end{abstract}

The main aim of this paper is to prove the following result.

THEOREM. Let $X$ be an arbitrary topological space and $\kappa>\omega$ a (regular) cardinal. If $w(Y)<\kappa(\pi(Y)<\kappa)$ holds whenever $Y \subset X$ and $|Y|<\kappa$ then $w(X)<\kappa(\pi(X)$ $<\boldsymbol{\kappa})$.

In [2] M. G. Tkačenko proved this (with $w$ only) for $T_{3}$ spaces and raised the question whether $T_{3}$ could be replaced by $T_{2}$. As we see, actually no separation axiom is needed.

We start to prove our theorem by establishing a lemma which might be of some interest in itself. We shall need the following piece of notation in stating it and also later. For an arbitrary set $X$, a family $\mathcal{S}$ of subsets of $X$ and $Y \subset X$ we put $\mathcal{S} \uparrow Y=\{S \cap Y: S \in \mathcal{S}\}$, the trace of $\mathcal{S}$ on $Y$.

LEMMA. Let $X$ be an arbitrary topological space and $\kappa>\omega$ be a regular cardinal. Moreover let $\left(Y_{\alpha}: \alpha \in \kappa\right)$ be an increasing sequence of subspaces of $X$ (i.e. $Y_{\alpha} \subset Y_{\beta}$

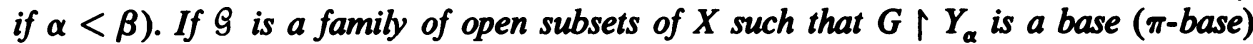

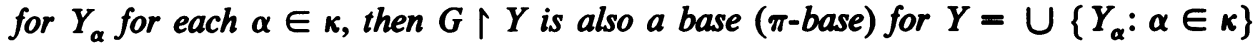
provided that $X$ contains no left-separated subspace of cardinality $\kappa$ (or equivalently, every subspace of $X$ has a dense subset of cardinality less than $\kappa, c f$. [1]).

Proor. We shall give the proof for the case of a base only, since that of the $\pi$-base is completely analogous. Suppose, on the contrary, that $\mathcal{G} \uparrow$ is not a base for $Y$. Then there is a point $p \in Y$ and a neighbourhood $U$ of $p$ such that if $p \in G \in \mathcal{G}$ then $G \cap Y \notin G \cap U$. Now we select by transfinite induction members $G_{\nu} \in \mathcal{G}$ and points $q_{\nu} \in Y \cap G_{\nu} \backslash U$ as follows. Assume $\mu<\kappa$ and $G_{\nu}, q_{\nu}$ have already been selected for $\nu<\mu$. Since $\kappa$ is regular we can find an ordinal $\alpha_{\mu}<\kappa$ such that $p \in Y_{\alpha_{\mu}}$ and $q_{\nu} \in Y_{\alpha_{\mu}}$ for every $\nu<\mu$. By our assumption there is a $G_{\mu} \in \mathcal{G}$ such that $p \in G_{\mu} \cap Y_{\alpha_{\mu}} \subset U$. Then we can pick a point $q_{\mu} \in G_{\mu} \cap Y \backslash U$. It is clear from our construction that if $\nu<\mu<\kappa$ then $q_{\nu} \notin G_{\mu}$; consequently the

Received by the editors May 3, 1979 and, in revised form, June 18, 1979.

AMS (MOS) subject classifications (1970). Primary 54A25.

Key words and phrases. Weight (z-weight) of a topological space.

(C) 1980 American Mathematical Society $0002-9939 / 80 / 0000-0382 / \$ 01.50$ 
sequence $\left\{q_{\nu}: \nu \in \kappa\right\}$ is left-separated, a contradiction.

Proof of THe Theorem. Again we restrict ourselves to the case of the weight function $w$, as that of $\pi$ is done similarly. Moreover, we first assume that $\kappa$ is regular. Our proof in this case is indirect, i.e. we assume $w(X)>\kappa$.

Then we define by transfinite induction subspaces $Y_{\alpha} \subset X$ and families of open sets $\mathscr{B}_{\alpha}$ with $\left|Y_{\alpha}\right|<\kappa$ and $\left|\mathscr{B}_{\alpha}\right|<\kappa$ for $\alpha<\kappa$ in the following way. Suppose that $\alpha<\kappa$ and $Y_{\beta}, \mathscr{B}_{\beta}$ have been defined for each $\beta<\alpha$. If $\alpha$ is limit (or 0 ) we put $Y_{\alpha}=\cup\left\{Y_{\beta}: \beta<\alpha\right\}$, and $\mathscr{B}_{\alpha} \supset \cup\left\{\mathscr{B}_{\beta}: \beta<\alpha\right\}$ is chosen in such a way that $\mathscr{B}_{\alpha} \uparrow Y_{\alpha}$ is a base for $Y_{\alpha}$ and $\left|\mathscr{T}_{\alpha}\right|<\kappa$. This is possible because $\left|Y_{\alpha}\right|<\kappa$ by the regularity of $\kappa$. Now, if $\alpha=\beta+1$, by our indirect assumption $\mathscr{B}_{\beta}$ is not a base for $X$, hence we can find a point $p^{(\beta)} \in X$ and its neighbourhood $U$ in such a way that no $B \in \mathscr{B}_{\beta}$ satisfies $p^{(\beta)} \in B \subset U$. Let us put $\mathscr{B}_{\beta}^{*}=\left\{B \in \mathscr{B}_{\beta}: p^{(\beta)} \in B\right\}$, then we can choose for each $B \in \mathscr{B}_{\beta}^{*}$ a point $q_{\beta} \in B \backslash U$. Finally, we put $Y_{\alpha}=Y_{\beta} \cup$ $\left\{p^{(\beta)}\right\} \cup\left\{q_{\beta}: B \in \mathscr{B}_{\beta}^{*}\right\}$ and $\mathscr{B}_{\alpha} \supset \mathscr{B}_{\beta}$ is chosen again so that $\mathscr{B}_{\alpha} \uparrow Y_{\alpha}$ is a base for $Y_{\alpha}$ and $\left|\mathscr{B}_{\alpha}\right|<\kappa$. Let us note that then $\mathscr{B}_{\beta} \uparrow Y_{\beta+1}$ is not a base for $Y_{\beta+1}$. Having completed the induction we put

$$
Y=\cup\left\{Y_{\alpha}: \alpha \in \kappa\right\} \text { and } \mathscr{B}=\bigcup\left\{\mathscr{B}_{\alpha}: \alpha \in \kappa\right\} \text {. }
$$

Now, observe that $w(Z)<\kappa$ for each $Z \subset X,|Z|<\kappa$ implies $d(Z)<\kappa$ for each such $Z$; consequently the conditions of our lemma are satisfied with the sequence of subspaces $\left\langle Y_{\alpha}: \alpha \in \kappa\right\rangle$ and the open family $\mathscr{B}$. Therefore $\mathscr{B} \uparrow Y$ forms a base for $Y$. But $|Y|<\kappa$; hence by our assumption $w(Y)<\kappa$ as well. Consequently, as is well known, we can select a subfamily $\mathcal{C} \subseteq$ with $|\mathcal{C}|=w(Y)<\kappa$ such that $\mathcal{P} Y$ is already a base for $Y$. Since $\kappa$ is regular we must have then an $\alpha<\kappa$ with $\mathcal{C} \subset \mathscr{B}_{\alpha}$. But, by our construction, $\mathscr{B}_{\alpha} \uparrow Y_{\alpha+1}$ is not a base for $Y_{\alpha+1}$ and, a fortiori, $\mathscr{B}_{\alpha} \uparrow Y$ is not a base for $Y$, a contradiction. This completes the proof for $\kappa$ regular.

Now let us consider the case in which $\kappa$ is singular. We claim that then there is a cardinal $\lambda<\kappa$ such that actually $w(Y)<\lambda$ holds whenever $Y \subset X$ and $|Y|<\kappa$. Assume, on the contrary, that no such $\lambda$ exists. Then we can find for each cardinal $\lambda<\kappa$ a subspace $Y_{\lambda} \subset X$ with $\left|Y_{\lambda}\right| \leqslant \kappa$ and $w\left(Y_{\lambda}\right)>\lambda$. But putting $Y=$ $\cup_{\lambda<\kappa} Y_{\lambda}$, we would have then $|Y| \leqslant \kappa$ and $w(Y) \geqslant \kappa$ (since $\kappa$ is a limit cardinal), which is impossible.

Now take any regular $\lambda<\kappa$ as in our claim. Then we can apply the first half of our proof to this $\lambda$ to conclude that $w(X)<\lambda<\kappa$.

The reader should notice that, since the $\pi$-weight is not monotone for subspaces, the second half of our proof (for $\kappa$ singular) cannot be applied to it. Thus e.g. the following problem remains open.

Problem. Does there exist a topological space $X$ such that $\pi w(Y)<\aleph_{\omega}$ whenever $Y \subset X$ and $|Y|<\kappa_{\omega}$ but $\pi w(X)>\kappa_{\omega}$ ?

\section{REFERENCES}

1. I. Juhász, Cardinal functions in topology, Math. Centre Tracts, No. 34, Math. Centrum, Amsterdam, 1971.

2. M. G. Tkačenko, Chains and cardinals, Dokl. Akad. Nauk SSSR 239 (1978), 546-549.

Mathematical Institute, Hungarian Academy of Sciences, 1053 Budapest V, Reáltanoda U. 13-15 HUNGARY 